\title{
Removal of Silicone Oil-Rewards and Penalties
}

\author{
W. A. FRANKS and P. K. LEAVER \\ London
}

\section{Summary}

Silicone oil is a useful tool in retinal reattachment surgery in selected cases, ${ }^{1-4}$ but complications, particularly cataract, glaucoma and keratopathy, have led to worries about its use for prolonged internal tamponade. ${ }^{5-8}$ Removal of silicone oil has been recommended to preempt or reverse these complications. A retrospective review of 120 eyes in which temporary silicone oil tamponade had been employed is presented. One hundred and twelve eyes were examined six months and two years after removal of silicone oil. Retinal redetachment occurred in 21 eyes $(19 \%)$.

Cataract formation was delayed by early removal of silicone oil, but after two years the majority of eyes had undergone surgery for cataract or had developed lens opacities.

Removal of silicone oil was ineffective in reversing an established pressure rise in nearly all cases. Three phakic eyes developed glaucoma after removal of silicone oil but no new cases occurred in phakic eyes in the two years following silicone oil removal.

Keratopathy was uncommon and was arrested and occasionally reversed by silicone oil removal.

Visual acuities improved in the majority of eyes after removal of silicone oil.

\section{Patients and Methods}

A consecutive series of 120 eyes of 116 patients who had undergone removal of silicone oil following successful retinal reattachment surgery from 1982 to 1987 were reviewed. Eyes with proliferative diabetic retinopathy were excluded.

All eyes had been treated with injection of 1000 cs silicone oil. Removal of silicone oil was performed under general or retrobulbar anaesthesia. An infusion cannula was inserted through a pars plana sclerotomy in the inferotemporal quadrant, $4 \mathrm{~mm}$ behind the limbus in phakic eyes and $3.5 \mathrm{~mm}$ in aphakic eyes. In phakic eyes a second pars plana sclerotomy was made superotemporally and silicone oil exchanged for balanced salt solution from the infusion cannula. In aphakic eyes silicone oil was removed through a $3 \mathrm{~mm}$ circumferential limbal incision. Silicone oil removal was combined with other procedures in 22 eyes.

Postoperatively topical antibiotics and corticosteroids were administered four times daily for two weeks. All eyes were examined by slit lamp biomicroscopy and indirect ophthalmoscopy on the first postoperative day, at one week, four weeks, three months, six months and two years postoperatively and more frequently if necessary. Intraocular pressure measurements were by Goldmann applanation tonometry.

Sixty nine left eyes were treated and 51 right eyes. The average age of patients was 41.5 years (range 12-81 years). 
Table I Effect of removal of silicone oil on the development of cataract: analysis of 60 phakic eyes with attached retina at 2 years (excluding eyes which underwent reinjection of silicone oil)

\begin{tabular}{lcccr}
\hline Lens status & \multicolumn{4}{c}{ Duration of intraocular silicone } \\
\hline $\begin{array}{l}\text { At time of silicone oil removal } \\
\text { phakic }\end{array}$ & $<6 / 52$ & $6 / 52-12 / 52$ & $>12 / 52$ & p-value \\
cataract & 19 & 26 & 15 & $<0.001$ \\
$\begin{array}{l}\text { Six months after silicone oil removal } \\
\text { cataract surgery within 6 months } \\
\text { clear lens }\end{array}$ & 4 & 11 & 9 & 0.128 \\
phakic & 4 & 9 & 9 & 0.06 \\
$\begin{array}{l}\text { Two years after silicone oil removal } \\
\text { cataract surgery after two years }\end{array}$ & 15 & 4 & 1 & 0.13 \\
clear lens & 10 & 17 & 6 & $<0.05$ \\
phakic & 4 & 17 & 11 & 0.50 \\
& 9 & 3 & 4 & 0.35 \\
\hline
\end{tabular}

*p-value for silicone oil removal at less than 12 weeks compared to greater than 12 weeks.

The duration of intraocular silicone oil ranged from three weeks to five years, with a mean of 30 weeks.

Eight patients failed to complete two years follow-up, leaving 112 eyes for analysis at six months and two years following removal of silicone oil.

The indications for use of silicone oil were giant retinal tear (57 eyes), giant retinal dialysis (one eye) and posterior breaks (16 eyes) and proliferative vitreoretinopathy (46 eyes).

\section{Results}

\section{Perioperative Complications of Silicone Oil Removal}

Hyphaema occurred following silicone-oil removal in 12 eyes and vitreous haemorrhage in ten. All haemorrhages cleared spontaneously without requiring further surgical intervention.

Hypotony occurred in 27 eyes in the first two weeks after surgery. Two eyes with attached retinae had intraocular pressures less than $8 \mathrm{mmHg}$ at six months and two years.

One suffered expulsive haemorrhage at the time of surgery and a second occurred in the eye undergoing Molteno tube implant two weeks after removal of silicone oil. Retinal redetachment was noted at the time of silicone oil removal in seven eyes.

\section{Postoperative Complications}

\section{Retinal Redetachment}

Retinal redetachment occurred in ten eyes in the first six months after silicone oil removal in addition to the seven eyes in which the retina was seen to redetach peroperatively. Thirteen of these 17 eyes underwent successful reattachment surgery. Between six months and two years, four further redetachments occurred, related to reproliferation of membranes. Surgery was successful in reattaching the retina in only one of these eyes.

Twelve eyes required further injection of silicone oil and two intraocular gas injection, while the retina remained detached in seven.

\section{Cataract: (Table I)}

The incidence of cataract was analysed in three groups according to the duration of intraocular silicone; five weeks or less, six to 12 weeks and greater than 12 weeks, to see if shorter duration of silicone oil tamponade was associated with a reduction in the number of eyes developing cataract. There was a high incidence of cataract in all three groups with no statistical difference between the number of eyes developing cataract at six months and two years. There was a trend, however, towards slower development of cataract the earlier silicone oil was removed and the lens was significantly more likely to be retained at six months if silicone oil was removed before 12 weeks. After two years most eyes had developed cataract.

Glaucoma: (Table II)

Raised intraocular pressure requiring medical or surgical treatment was present in ten phakic eyes and 18 aphakic eyes at the time of removal of silicone oil. After two years no further phakic eyes had developed glaucoma 
Table 2 Analysis of potential risk factors for glaucoma in eyes with temporary silicone oil tamponade

\begin{tabular}{lccc}
\hline & Total & Glaucoma at 2 years & $p$-value \\
\hline Aphakia & 39 & 21 & $<0.001$ \\
Raised IOP at time of oil removal & 27 & 24 & $<0.001$ \\
Giant tear & 51 & 17 & 0.02 \\
PVR & 45 & 11 & NS \\
Post break & 15 & 3 & NS \\
Scleral buckle & 79 & 23 & NS \\
\hline
\end{tabular}

but three additional aphakic eyes commenced treatment for raised intraocular pressure.

Aphakia was a significant risk factor for the presence of glaucoma at the time of silicone oil removal $(\mathrm{p}<0.001)$. Of 18 eyes which underwent lensectomy at the time of silicone oil injection, ten required treatment for glaucoma after two years $(55 \%)$ and of 18 eyes aphakic prior to silicone oil injection, 11 $(61 \%)$ required treatment for glaucoma two years after silicone oil removal, a similar proportion in each group.

In aphakic eyes there was a trend towards an increase in the incidence of glaucoma with duration of intraocular silicone. The mean duration of intraocular silicone was 75 weeks in eyes with glaucoma compared to 58 weeks in those not developing this complication, but the numbers were small, and did not reach statistical significance.

The number of previous retinal procedures, type of retinal detachment, and the use of scleral buckling did not correlate significantly with the subsequent development of glaucoma.

\section{Keratopathy}

Keratopathy occured in five aphakic eyes. In one, corneal opacification reversed after silicone oil removal and one underwent penetrating keratoplasty at the time silicone oil was removed. In two, keratopathy remained stable and one developed bullous keratopathy.

\section{Advantages of Silicone Oil Removal}

Visual Acuity: (Fig. 1)

Mean best corrected visual acuity at the time of silicone oil removal was $6 / 60$, improving to 6/36 at six months and maintained at two years. In 30 eyes visual acuity was $6 / 18$ or better after two years.
If eyes with retinal redetachment are excluded, visual acuity improved in 52 eyes, worsened in 20 and was unchanged in 19. Improvement in vision was associated with cataract extraction, continued improvement in retinal function after successful retinal reattachment, removal of silicone oil emulsion and reduction in the optical effects of the silicone oil bubble. Deterioration of vision was related to development of cataract, glaucoma, macular pucker and keratopathy.

\section{Discussion}

Visual acuity following silicone oil removal was improved in the majority of eyes in this series and in 30 , visual acuities were $6 / 18$ or better. This level of vision was useful to many binocular patients and temporary silicone-oil tamponade may be of benefit to patients with good vision in the fellow eye. Removal of silicone oil exerts some beneficial effects by abolishing variable vision associated with movement of the silicone oil bubble and reducing visual disturbance caused by emulsion. ${ }^{9}$ Many eyes underwen ${ }^{+}$conventional extracapsular cataract surgery with intraocular lens implantation. Removal of silicone is advantageous in these cases as it allows accurate biometry, reduces capsular opacification and abolishes the variable optical effects caused by the silicone oil bubble.

Silicone oil removal was associated with retinal redetachment in $19 \%$ of eyes. This is a similar finding to that in other series. ${ }^{5-8}$ In most cases the retina was reattached but in seven eyes it remained detached. Redetachment of the retina also occurs in eyes where silicone oil has not been removed, usually as a result of reproliferation of membranes. In such eyes the recruitment of subretinal fluid may be limited by silicone oil and macular vision may be spared but if silicone oil is 


\section{Visual acuity after silicone oil removal (91 eyes with attached retinae)}

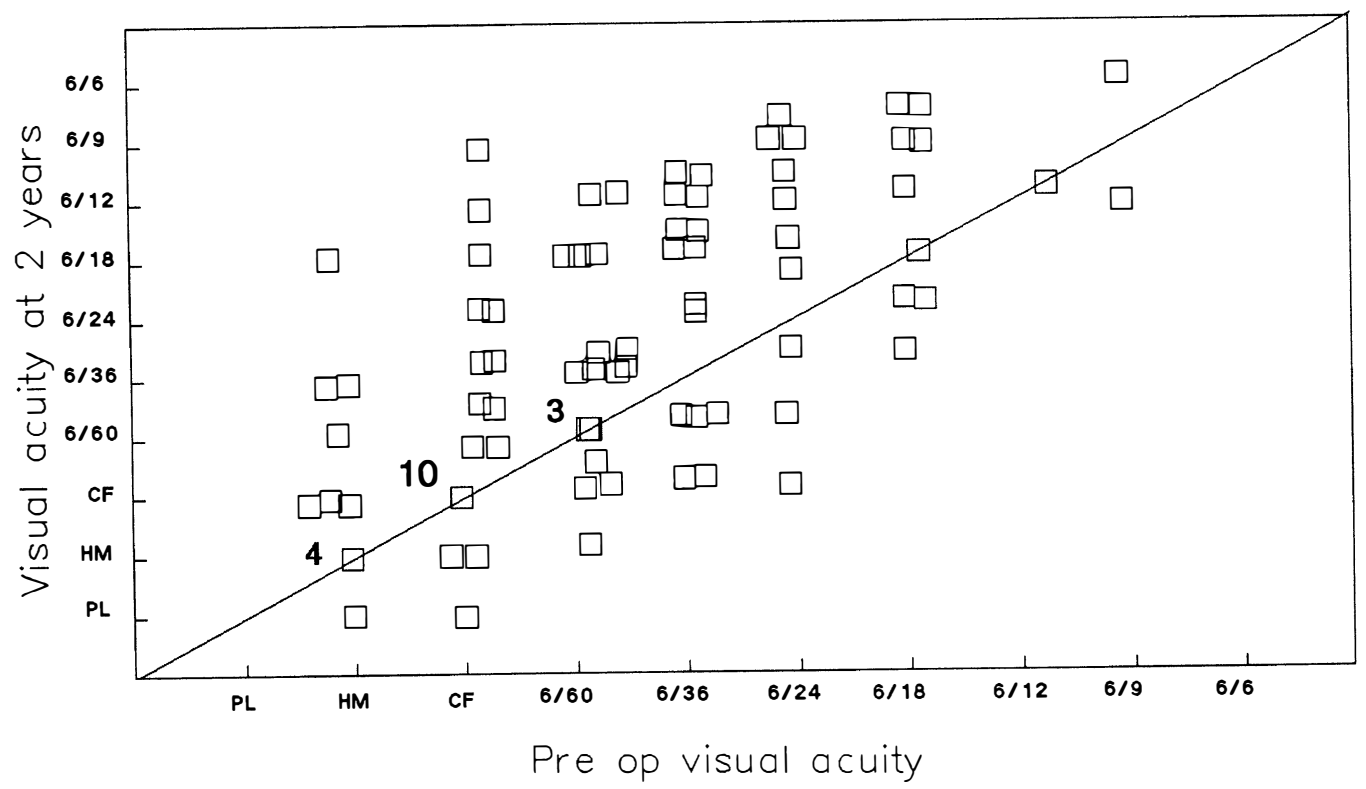

Fig 1. Visual acuity after silicone oil removal (91 eyes with attached retinae).

removed, redetachment is likely to be rapid and extensive and urgent surgical reintervention is required.

Early removal of silicone oil was associated with a trend towards a reduction in cataract formation and the lens was retained in significantly more eyes from which silicone oil was removed after less than 12 weeks than in those in which the duration of intraocular silicone was longer. It appears that early removal of silicone oil delays the development of cataract but does not prevent it.

Glaucoma was common in this series, $23 \%$ of eyes undergoing treatment for raised intraocular pressure prior to silicone oil removal. Removal of silicone oil was ineffective in reversing an established pressure rise in all but one eye. After silicone oil removal no further phakic eyes developed glaucoma despite the presence of persistent emulsion in the anterior chamber drainage angle. Three aphakic eyes developed glaucoma in the two year follow-up period, probably reflecting the more severe anterior segment disturbance these eyes had suffered.
The principal risk factors for the development of glaucoma were aphakia and raised intraocular pressure at the time of silicone-oil removal. Following Ando's report ${ }^{13}$ in 1985, an inferior iridectomy was employed in all aphakic eyes undergoing silicone oil injection. This reduced the number of eyes developing angle closure glaucoma with pupil block, and the number of eyes with peripheral anterior synechiae and chronic angle closure subsequently requiring drainage surgery. A few cases of acute glaucoma continued to occur, however, when iridectomies became blocked by fibrin. ${ }^{14}$

Scleral buckling has been implicated in causing acute pressure rise following vitrectomy, ${ }^{15}$ but we found no evidence of its influence on the incidence of glaucoma six months and two years after removal of silicone oil.

The incidence of glaucoma was high compared to other series. ${ }^{5,7,8}$ The purity of the silicone oil used in different centres varies considerably ${ }^{16}$ and the use of 1000 centistoke oils with a high proportion of small molecular weight components may contribute to emulsi- 
fication and damage to the anterior chamber drainage angle. Higher viscosity silicone oils have less impurities but are more difficult to remove from the eye. Further studies are needed to assess if their use is associated with a lower incidence of complications.

\section{Conclusions}

An improvement in visual acuity and visual function follows removal of silicone oil, provided that the retina remains attached.

Most eyes develop cataracts but in eyes in which silicone oil is removed at less than 12 weeks their development is delayed.

Glaucoma may be prevented by early removal of silicone oil but once a pressure rise is established, removing silicone oil will not reverse it.

Keratopathy in aphakic eyes can occasionally be reversed or arrested by removal of silicone oil.

Removal of silicone oil is recommended to improve visual potential and reduce the risks of sight threatening complications in eyes in which the retina has been successfully reattached.

Wherever possible it is recommended that silicone oil be removed within 12 weeks of injection.

Key words: Cataract, Glaucoma, Internal Tamponade, Keratopathy, Retinal detachment, Silicone oil.

\section{References}

${ }^{1}$ Cibis PA, Becker B, Okun E, Canaan S: The use of liquidsilicone in retinal detachment surgery. Arch Ophthalmol 1962, 68: 590-9.

${ }^{2}$ Scott JD: Treatment of massive vitreous retraction. Trans Ophthalmol Soc UK 1975, 95: 429.

${ }^{3}$ McCuen BW, Landers MD, Machemer R: The use of silicone oil following vitrectomy for retinal detachment with advanced proliferative vitreoretinopathy. Ophthalmology 1985, 92: 1029.

${ }^{4}$ Leaver PK, Grey RHB, Garner A: Silicone oil in the treatment of massive preretinal retraction.11. Late complications in 93 eyes. Br J Ophthalmol 1979, 63: 361.

${ }^{5}$ Chan C, Okun E: The question of ocular tolerance to intravitreal liquid silicone. A long-term analysis. Ophthalmology 1986, 93: 651-9.

${ }^{6}$ Gonvers M: Temporary silicone oil tamponade in the management of retinal detachment with proliferative vitreoretinopathy. Am J Ophthalmol 1985, 100: 239.

${ }^{7}$ Casswell AG and Gregor ZJ: Silicone oil removal. 11. Operative and postoperative complications. Br J Ophthalmol 1987, 71: 898.

${ }^{8}$ Zillis JD, McCuen BW, De Juan E, Steffansson E, Machemer R: Results of silicone oil removal in advanced proliferative vitreoretinopathy. Am J Ophthalmol 1989, 108: 15-21.

${ }^{9}$ Stefansson E and Tiedeman J: Optics of the eye with air or silicone oil. Retina 1988, 8: 10 .

${ }^{10}$ Leaver PK, Cooling RJ, Feretis EB, Lean JS, McLeod D: Vitrectomy and fluid/silicone exchange for giant retinal tears: results at six months. Br J Ophthalmol 1984, 68: 432-8.

${ }^{11}$ Billington BM and Leaver PK: Vitrectomy and fluid/ silicone oil exchange for giant retinal tears: results at 18 months. Graefe's Arch Clin Exp Ophthalmol 1986, 224, 7-10.

${ }^{12}$ Leaver PK and Billington BM: Vitrectomy and fluid/ silicone exchange for giant retinal tears: 5 years follow-up. Graefes Arch Ophthalmol 1989, 227: 323-7.

${ }^{13}$ Ando F: Intraocular hypertension resulting from pupillary block by silicone oil. Am J Ophthalmol 1985, 99: 87.

${ }^{14}$ Laganowski $\mathrm{H}$ and Leaver PK: Silicone oil in the aphakic eye: the influence of a six o'clock peripheral iridectomy. Eye 1989, 3: 338-48.

${ }^{15}$ Han DP, Lewis H, Lambrou FH, Mieler WF, Hartz A: Mechanism of intraocular pressure elevation after pars plana vitrectomy. Ophthalmology 1989 , 96: 1354-62.

${ }^{16}$ Parel JM: Silicone oils: physicochemical properties. In Retina. Ryan S, ed. Volume 3. Surgical Retina. Glaser BM, Michels RG, eds. CV Mosby and Co. 1989. Ch. 127, p 263-266. 